\title{
Single-particle cryo-EM studies of ERp44-ERAP1 and ERp44-ERAP2 reveal their ER-retention mechanism and structural dynamics involved
}

\author{
R Arya ${ }^{1}$, L Stern ${ }^{1}$ \\ ${ }^{1}$ UMass Medical School, Worcester, MA \\ richa.arya@umassmed.edu
}

ERAP1 is an ER-resident aminopeptidase with roles in antigenic peptide processing for MHC-I presentation and angiotensin processing for blood pressure control. Previous studies had implicated ERp44 C29 in the thioredoxinlike a-domain and ERAP1 C498 in the domain-II "exon-10" loop as important in the ER-retention mechanism. We wanted to investigate interactions between ERp44 and ERAP1 beyond the C29-C498 disulfide that might contribute to the specificity and regulation of complex formation, and to determine whether ERAP2, a paralog of ERAP1 that also generates peptides for antigen presentation, could utilize a similar mechanism for ER retention. Using purified recombinant ERp44 and ERAP1, we confirmed that the C29-C498 intermolecular disulfide forms spontaneously upon mixing ERp44 and ERAP1, resulting in a 1:1 complex. ERp44 interaction with ERAP was not pH-sensitive $(5.7-7.7)$, unlike its interaction with other client proteins, suggesting a possible novel mode of interaction. Using cryo-EM we obtained 4.4Å resolution maps for the ERp44-ERAP1 complex. The 3-D volumes contain both closed and semi-open conformations of ERAP1 though, suggesting that the ERp44 interaction might stabilize the less open form of the protein, and reveals substantial domain interactions between the two proteins. We are examining this structure to understand the structural basis for the preferential reactivity of ERp44 C29 over C63 and ERAP1 C498 over C486, and to gain insight into the mechanism of release in the ER. Like ERAP1, ERAP2 is ER-resident, but the retention mechanism is unknown. We observed a similar interaction of ERp44 with ERAP2 as with ERAP1, with ERp44 C29 forming an intermolecular disulfide with ERAP2 C514. Cryo-EM 3D volumes for the ERp44-ERAP2 complex at 6.0 ̊ reveal ERAP2 in mostly open conformation. These results suggest that ERAP2 is retained within ER by a similar mechanism as ERAP1 but in a different conformation as ERAP1. This might propose different conformational dynamics of ERAP2 as ERAP1 on interaction with ERp44.

Acta Cryst. (2020). A76, a138 